\title{
Rapid eye movement behaviour disorder
}

Richard G Weeks BAppSc, BMBS, FRANZCP

PSychiatrist and Senior Lecturer ${ }^{2}$

\section{Jason A D'Costa} MBBS, FRACP Sleep Physician ${ }^{\top}$

Vinod Aiyappan MD, MRCP, FRACP Respiratory and Sleep Physician ${ }^{1}$

Ching Li Chai-Coetzer MBBS, FRACP, PhD Sleep and Respiratory Physician ${ }^{1}$

Nick A Antic MBBS, FRACP, PhD Clinical Director ${ }^{1}$

1 Adelaide Institute for Sleep Health, Repatriation General Hospital, Adelaide, SA.

2 School of Medicine, Flinders University, Adelaide, SA.

Richard.Weeks@ health.sa.gov.au

doi: 10.5694/mjal3.11000

\section{Clinical record}

A 70-year-old man was referred to a specialist sleep service in December 2010 by his general practitioner at the prompting of his wife. He reported a 7-8-year history of near-nightly, increasingly severe sleep disturbance with vivid dreams which would occur in the latter half of the night. Bedclothes were often found strewn around the room, and he would wake in unusual positions. The dreams usually related to being chased or attacked, and the patient could recall them in great detail. While asleep, the patient frequently shouted in response to the dreams. Violent motor responses to the dreams were also common - the patient had kicked, punched and attempted to choke his wife while asleep, he had fallen out of bed, and he had leapt into wardrobes and onto bedside tables. The patient and his wife had suffered injuries. He had surrounded his bed with mattresses to prevent further injury, and had taken to restraining himself to the frame of his bed with two belts strapped firmly around his waist and chest. He reported being aware of the violent behaviour. His wife had moved into a separate bedroom for her own safety.

The patient reported that he slept for 6-7 hours/night and did not experience problematic daytime sleepiness (his Epworth Sleepiness Scale score was 1 out of a possible 24 [a normal daytime sleepiness level]). He did not have a history of any of the following: snoring, witnessed apnoeas, choking arousals or other symptoms suggestive of obstructive sleep apnoea; symptoms of restless legs syndrome; cataplexy, hypnagogic hallucinations or sleep paralysis: or psychiatric illness or neurological problems. He reported taking oxazepam 15-30 mg on rare occasions, but this had no impact on the sleep disturbance. His body mass index was $36 \mathrm{~kg} / \mathrm{m}^{2}$. Results of a physical examination, including a full neurological examination, were unremarkable. The patient was not taking any psychotropic medication. Results of blood tests (complete blood count; levels of electrolytes, urea, creatinine and C-reactive protein; liver function tests; thyroid function tests; and iron studies) were unremarkable.

The patient underwent overnight laboratory-based polysomnography (Figure) with video monitoring. Total sleep time was 404 minutes, with a normal sleep efficiency (time asleep $\div$ time in bed) of $85.7 \%$, a normal arousal index of 9.3 events/hour and a normal apnoeahypopnoea index of 0.7 events/hour. Minor periodic limb movements were detected but were not associated with electroencephalographic arousals.

During periods of rapid eye movement (REM) sleep, abnormal motor activity was detected (Figure, B). Muscle tone, as measured by electromyographic tone, actually increased during some periods of REM sleep, and phasic limb movements were seen during these periods. The patient thrashed his arms around on a number of occasions during REM sleep, but no other violent motor activity was detected. There was no evidence of seizure activity on the electroencephalogram recording. The polysomnography findings were thought to be consistent with a diagnosis of REM sleep behaviour disorder.

After receiving education regarding his diagnosis, the patient was started on clonazepam $0.25 \mathrm{mg}$ at night. There was no significant response at this dose, which was then titrated up in $0.25 \mathrm{mg}$ increments to a dose of $1 \mathrm{mg}$ at night, at which point the symptoms abated significantly. At 2-year follow-up, the patient reported that this improvement had been maintained, with violent dream enactment behaviour occurring rarely, and no episodes of falling out of bed or injuries. He reported significant subjective improvement in his sleep quality. To date, no emergence of neurological symptoms has been noted.

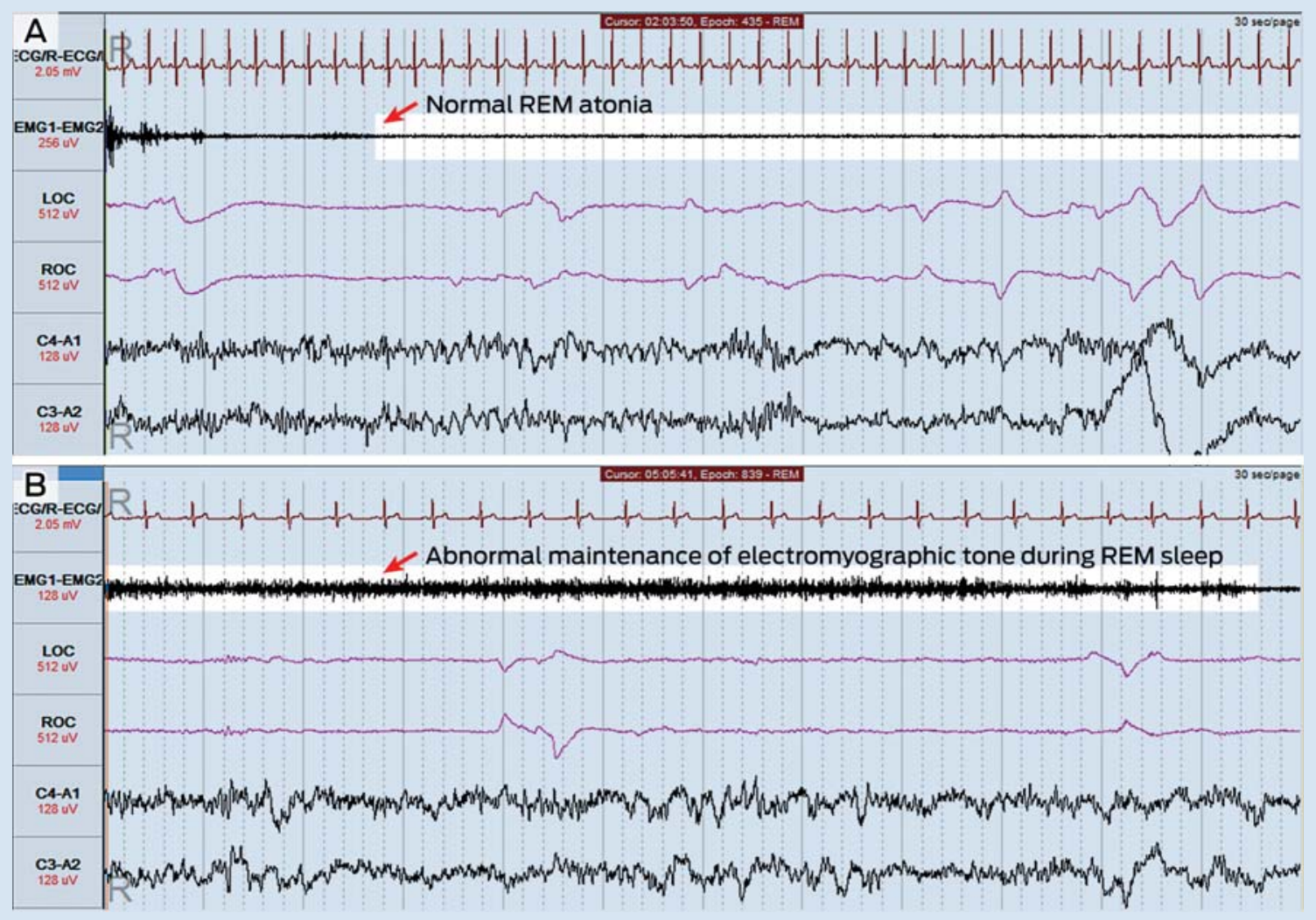

Polysomnography recordings showing normal atonia (A) and abnormal maintenance of electromyographic tone (B) during REM sleep. 


\section{Lessons from practice}

- Rapid eye movement behaviour disorder (RBD) is a rare but important sleep disorder that mainly affects men over 50 years of age. Significant sleep-related injury to the patient or bed partner is common.

- RBD is associated with the $\alpha$-synucleinopathies and may predate clinical emergence of these syndromes by many years.

- Diagnosis is made on the basis of the clinical history and supportive polysomnography findings. Polysomnography is also required to exclude other potential causes such as obstructive sleep apnoea.

- In most cases, RBD is highly responsive to low doses of clonazepam, and the therapeutic effect is usually maintained for a long period without the need for dose escalation.

apid eye movement behaviour disorder (RBD) is a parasomnia that emerges from the rapid eye movement (REM) stage of sleep, characterised by abnormal, usually violent and potentially injurious behaviour (vocalisations or motor activity) that occurs in response to dreams and mirrors dream content. ${ }^{1}$ The dream mentation acted out is usually threatening in nature and often vividly recalled; $33 \%-65 \%$ of patients report a sleep-related injury to themselves or their bed partner. ${ }^{2}$ RBD predominantly affects men over the age of 50 years, but males and females of all ages can be affected. 2,3 Events can occur with variable frequency, from multiple times every night to once every few months, and there is often a prolonged period between symptom onset and diagnosis. ${ }^{3,4}$

RBD can be idiopathic or associated with an underlying neuropathological condition, particularly the $\alpha$-synucleinopathies Parkinson disease, Lewy body dementia and multisystem atrophy. ${ }^{4} \mathrm{Up}$ to $65 \%$ of patients with RBD develop one of these conditions after a variable lag period, which is sometimes measured in decades. ${ }^{5}$ In addition, RBD is diagnosed in significant numbers of patients with a known $\alpha$-synucleinopathy. ${ }^{6}$ Arousals during REM sleep due to obstructive sleep apnoea can mimic features of RBD, making this an important differential diagnosis. ${ }^{7}$ Diagnosis of RBD requires suggestive clinical features and abnormalities detected on overnight polysomnography, particularly the maintenance of electromyographic tone during the REM stage of sleep and associated phasic limb movements.
Treatment of RBD is focused on reducing the frequency and severity of nightmares and associated abnormal behaviours, and limiting the potential for sleep-related injury. Management should involve environmental modification and pharmacotherapy. Clonazepam has the best evidence base for treating $\mathrm{RBD}^{8}$ - relatively low clonazepam doses are efficacious in most cases and, once symptomatic control of nocturnal behaviour has been achieved, the therapeutic effect is usually maintained for years and dose escalation is rarely needed. ${ }^{9}$ Mechanisms of action for most agents used to treat this condition are thought to involve suppression of tonic and/or phasic REM muscle activity. ${ }^{10}$

Acknowledgements: We thank Laura Jarema for her assistance in the production of the figure for this article.

Competing interests: Richard Weeks has received a Foundation Daw Park research grant. Ching Li Chai-Coetzer has received research funding from the National Health and Medical Research Council (NHMRC), Flinders Medical Centre Clinicians Trust and Flinders Medical Centre Foundation, and equipment donations from ResMed, Philips Respironics and SomnoMed. Nick Antic has received research funding from the NHMRC, Philips Respironics and Fisher and Paykel Healthcare, equipment donations from ResMed, Philips Respironics and SomnoMed, and lecture fees and payment for development of educational presentations from ResMed, Boehringer Ingelheim and GlaxoSmithKline.

1 American Academy of Sleep Medicine. International classification of sleep disorders: diagnostic and coding manual. 2nd ed. Illinois: AASM, 2005.

2 Boeve BF, Silber MH, Ferman TJ, et al. REM sleep behavior disorder and degenerative dementia: an association likely reflecting Lewy body disease. Neurology 1998; 51: 363-370.

3 Schenck CH, Hurwitz TD, Mahowald MW. Symposium: Normal and abnormal REM sleep regulation: REM sleep behaviour disorder: an update on a series of 96 patients and a review of the world literature. J Sleep Res 1993; 2: 224-231.

4 Olson EJ, Boeve BF, Silber MH. Rapid eye movement sleep behaviour disorder: demographic, clinical and laboratory findings in 93 cases. Brain 2000; 123: 331-339.

5 Gagnon JF, Postuma RB, Mazza S, et al. Rapid-eye-movement sleep behaviour disorder and neurodegenerative diseases. Lancet Neurol 2006; 5: 424-432.

6 Claassen DO, Josephs KA, Ahlskog JE, et al. REM sleep behavior disorder preceding other aspects of synucleinopathies by half a century. Neurology 2010; 75: 494-499.

7 Iranzo A, Santamaria J. Severe obstructive sleep apnea/hypopnea mimicking REM sleep behavior disorder. Sleep 2005; 28: 203-206.

8 Aurora RN, Zak RS, Maganti RK, et al. Best practice guide for the treatment of REM sleep behavior disorder (RBD). J Clin Sleep Med 2010; 6: 85-95.

9 Schenck CH, Mahowald MW. Polysomnographic, neurologic, psychiatric, and clinical outcome report on 70 consecutive cases with REM sleep behavior disorder (RBD): sustained clonazepam efficacy in $89.5 \%$ of 57 treated patients. Cleve Clin J Med 1990; 57 Suppl: S9-S23.

10 Gagnon JF, Postuma RB, Montplaisir J. Update on the pharmacology of REM sleep behavior disorder. Neurology 2006; 67: 742-747. 